\title{
Description of approaches to the study of decentralized complex systems in the management of the regional construction cluster
}

\author{
Dmitrii Anufriev' and Artem Holodov* \\ Astrakhan State University of Architecture and Civil Engineering, Department of CAD and modelling \\ systems, 414056, Astrakhan, Tatischeva str., Russia
}

\begin{abstract}
Regional construction cluster, which is considered as a decentralized socio-economic system, territorially localized within the region, consisting of interconnected enterprises of construction and related industries. Development of a method for centralized management of a complex socio-economic system based on the integration of simulation approaches. Approaches are considered that allow the transition from consideration of a decentralized complex system to the management of a centralized structure through the introduction of image agents and the formation of a multiphase queuing network, where the four-channel queuing system with expectations is considered as the phase. The structure of the simulation model is presented, which makes it possible to model the functionality of the regional construction cluster. The approaches to integration of the agent approach with modules of system dynamics and modules implementing the discrete-event approach for simulation of business pro-cesses in a complex socio-economic system that take into account the probabilistic structure of the emerging processes are considered in the article..
\end{abstract}

\section{Introduction}

Regional building cluster consists of a finite set of objects once-type personal and different functionality. In addition, stochastic (probabilistic) relationships can arise between objects. If we consider a regional building cluster from the viewpoint of a complex (generalized) object, then we can conclude that it is in a certain environment in which the effects on it (the object) are formed and appear, which are external to it [1]. In the same way, as a result of the arising connections between objects within the generalized object, internal influences arise, which in turn influence the environment. In fact, the system introduces a SUBSTANCE-AGENT, which has both a direct and a feedback. But this system can also be considered from the point of view of SUB-STANCE-ESSENCE, where ESSENCE includes agents of various types and in this case should be considered as some abstract

\footnotetext{
Corresponding author: artemhol@ rambler.ru
} 
structure having an artificial nature. But nevertheless, under the resulting external influence, this abstract structure (ESSENCE) should somehow be organized [2]. That is, the agents of different types must be organized, which actually constitute the essence itself. One can of course argue that a regional construction cluster is an open system, but we accept the fact that it is closed (closed) at the time interval under consideration. The only possibility to study the functioning of such a complex system and, in particular, to control it, is simulation modelling.

\section{Formulation of the problem}

It is necessary to develop generalized approaches to the development of an imitation model for the functioning of a regional building cluster, consisting of a finite set of agents of different types and the determination of the feasibility of performing work that arises in the external envi-ronment. According to this formulation of the problem, we are dealing with a decentralized com-plex system, since it is envisaged to use agents of different types and it is necessary to develop an external task structure that will be considered from the position of an additional agent with a unique structure. In addition, it is necessary to evaluate the possible level of modeling abstraction in accordance with the approaches and techniques used in constructing the simulation model $[3,4]$.

\section{The results and methods of research}

The development of the simulation model was carried out in the simulation system AnyLogic 8.3.2.

Immediately turn to the consideration of the fact that all enterprises and organizations that are part of the regional construction cluster (RCC) can be combined (clustered) on the basis of the structure of functioning, which will be discussed in more detail below. In particular, according to the results of the research in Astrakhan region, four groups of the main participants of the RCC were singled out [5]:

Power structures at the level of the region, municipalities;

1. Large enterprises of the region;

2. Enterprises interconnected with regional industry vendors;

3. Public structures.

In accordance with this fact, the structure of the developed simulation model will include four agents (Type_A, Type_B, Type_C, Type_D) having their populations (type_A, type_B, type_C, type_D). Moreover, different populations of intersection is equal to the empty set.

Now let's clarify the fact that the simulated complex system is decentralized and try to centralize it, that is, to define some organizational structure without changing the essence of the processes in discrete-event approach methods. The process of centralization leads to a complication of the structure of the model being developed, as well as to an increase in the level of abstraction: from operational to tactical. That is, it is necessary to envisage the implementation of additional steps, in order to improve the level of modeling abstraction. Here, of course, there are philosophical aspects associated with the transition from decentralization to centralization and with an assessment of the level of abstractions. Thus, the authors consider centralized complex systems from the positions of multiphase queuing networks (QN) [6], where each phase is considered from the point of view of queuing systems (QS) [7]. In addition, the fact that the use of priorities in absolute and relative priorities in QS leads to a reduction in the level of modeling abstraction [8]. 


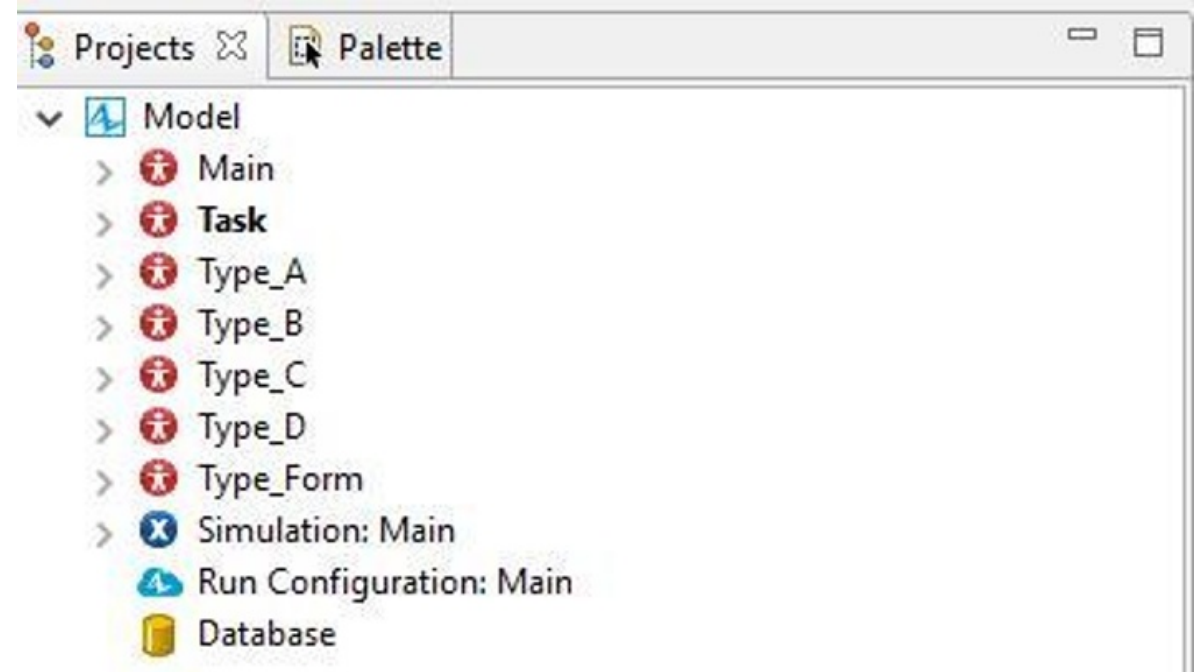

Fig. 1. Structure of the simulation model

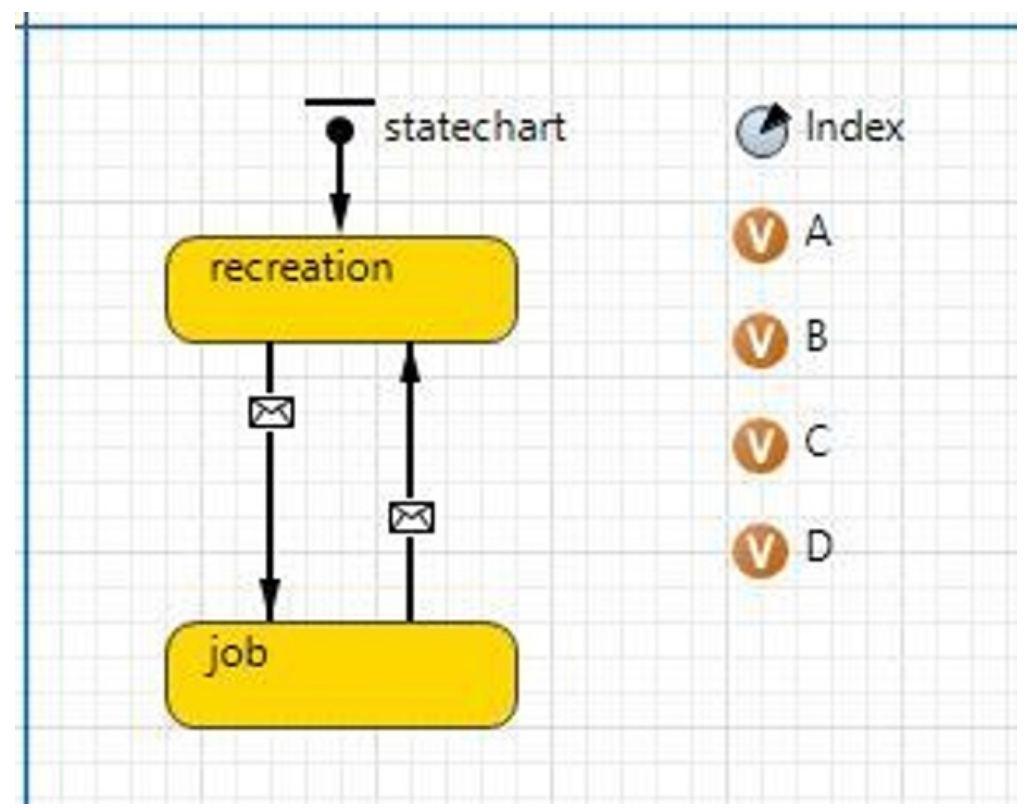

Fig. 2. Structure of Type_Form

Figure 1 shows the structure of the simulation model being developed. The Main agent is always present in the model and through it the connections between the agents entered are formed. The four agents described earlier (Type_A, Type_B, Type_C, Type_D) are present. An agent type Type_Form appeared, it is an agent-image that can participate in the flow diagram and that eliminates the problem of different types of agents $[9,10]$. Its structure is shown in Figure 2. The type_Form population is in the Main agent and its size is equal to the sum of all populations: type_A, type_B, type_C, and type_D. 


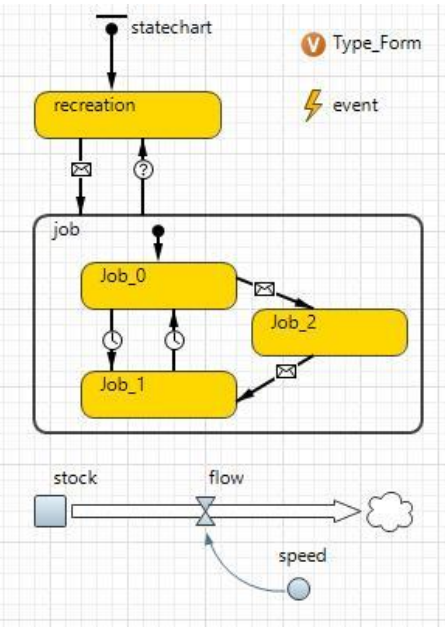

$\mathbf{a}$

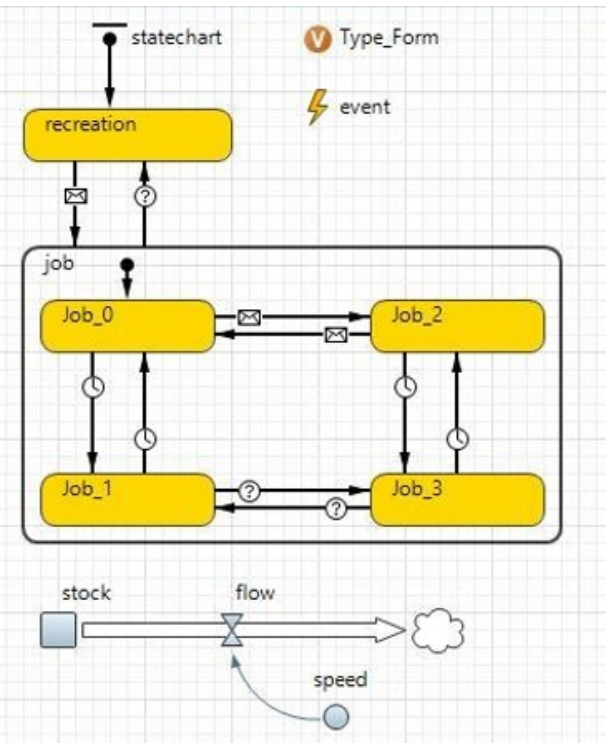

c
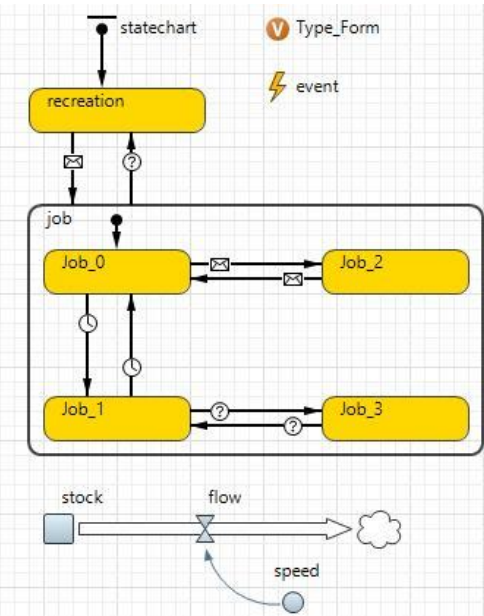

b
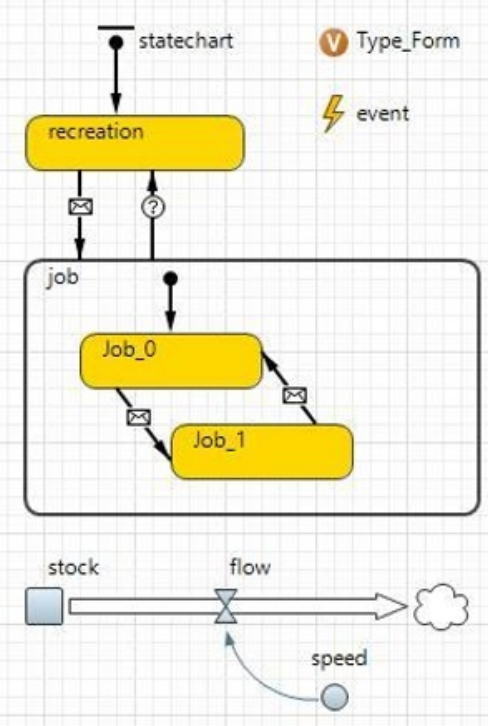

d

Fig. 3. Structure of agents of four types: $a-$ agent structure Type_A; b - agent structure Type_B; $c-$ agent structure Type_C; $d$ - agent structure Type_D.

It follows from Figure 2 that the Type_Form structure includes an Index parameter with an integer data type, four global variables $\mathrm{A}, \mathrm{B}, \mathrm{C}$ and $\mathrm{D}$, respectively having the types of agents (Type_A, Type_B, Type_C, Type_D) and the statechart state diagram defined by two states, possibly composite, and transitions between states that occur upon the arrival of specified messages.

Now let's look at the structures of the four agents (Type_A, Type_B, Type_C, Type_D), which mimic the functionality of the organizations entering the RCC (Figure $3 a, b, c, d$ ), here there are certain requirements inherent in all four types.

Reference to appropriate agent image is carried out by introducing the global variable Type_Form. The event event will determine the stochastic flow, in particular, if the time intervals are distributed according to the exponential distribution law, then the simplest flow is formed. The integrated event diagram has two composite recreation and job events. 
On the transitions between multiple event imposed certain requirements, namely the transition from recreation event to event job is done to obtain a specific message, and the transition from an event job to the recreation event is carried out under the condition. I would also like to note the fact that the compound event job simulates the functionality of the agent, this is done by introducing substages of the form job_i, where $\mathrm{i}$ takes, of course, the whole value and the number of them is limited. It is also possible to establish transitions between substates of all types that can be reached, namely: timeout transitions, condition transitions, and message retransmissions. In this regard, the possibility is feasible to achieve the operational level of abstraction modeling.

Also in the structure of these agents integrated module system din-Nomics [11, 12], including a storage stock, flow stream and flow-related dynamic variable speed. In analogy with the real world and the real situation, this module of system dynamics can be represented as follows: an organization receives an order for the performance of work, for any work there is a quantitative definition of the value in time (hours, days, weeks, etc.) this value is placed in the stock drive and with a certain constant speed, which is stored in the dynamic variable speed, begins to decrease. This process continues until the moment the value of the drive becomes zero and it is this condition that makes the transition of states in the event diagram - the work is over. Naturally, the use of a constant rate of performance of work leads to an idealization of the process and thus to an increase in the level of abstraction of the model. It is necessary to identify approaches that address this problem, and the approaches must be implemented by software, and they are [13,14]. They consist in the management of either a circular event Event, or in the definition of a table-defined function Table Function with the specification of nodal values with the next interpolation, or approximation. Now let's move on to the structure of the Task agent, which simulates the emergence of external influence of the SUBSTANCE (Figure 4). The external impact is considered as an order in the implementation of which various organizations and enterprises belonging to the RCC should take part.

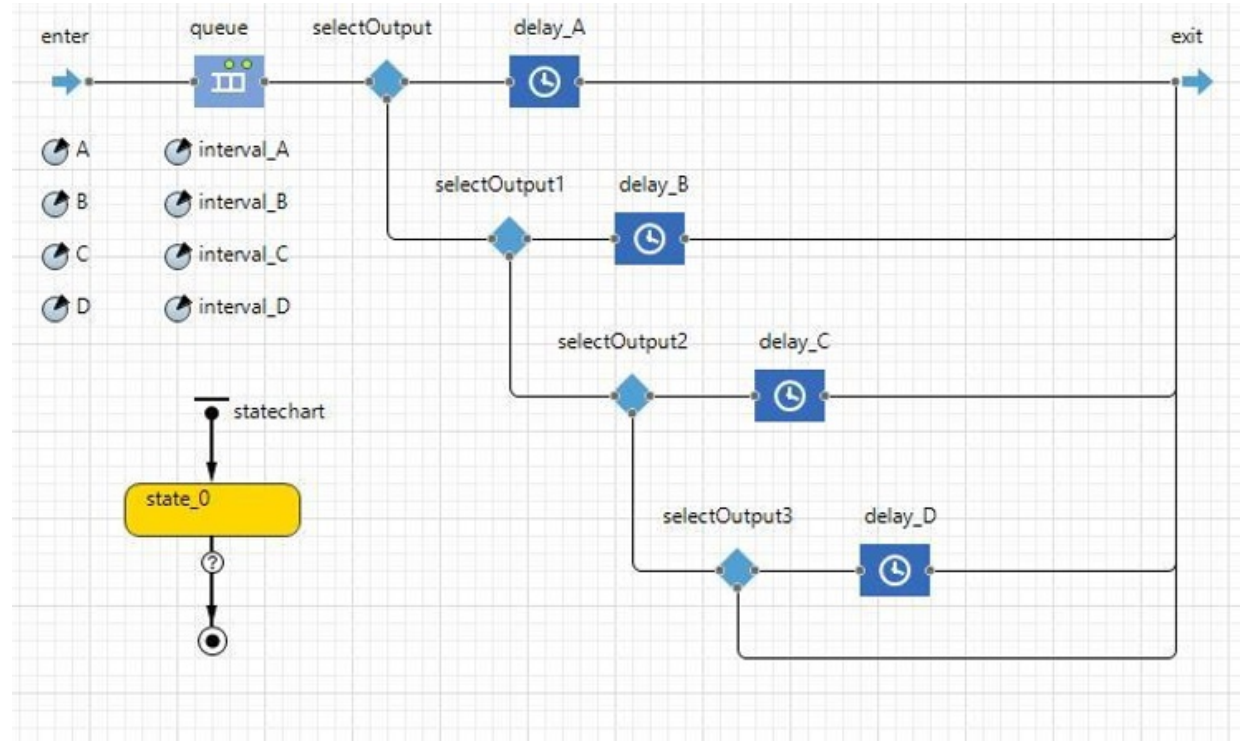

Fig. 4. Structure of the Task Agent

For any order there must be a technological map that determines the types and volumes of the necessary work, as well as the sequence that sets them (sequence), which in turn can be realized by means of an oriented graph. Any graph can be represented as a union of non- 
intersecting time sections, by examining the graph together with the time axis on which the time interval is selected, which in turn is divided into a certain number of intervals. In this article, the authors do not consider the problems associated with the development of algorithms for optimal partitioning into intervals, this will be done in the following papers. In the current study, we will consider that at any time interval it is necessary to envisage the possibility of participation of organizations of all four groups included in the RCC of the Astrakhan region. This is possible with the use of QS with waiting and four, parallelconnected, serving channels (devices) [15]. In the event that fewer agents (organizations) participate in the time interval, it is necessary to set the average service time in the channel to zero, or a very high frequency of service in the exponential distribution. Based on all of the above, the structure of the Task agent for the time section was developed. Combining all time sections to be implemented by the dynamical structure of the XYZ collection (java), having a static-dimensional array type double [4]. Accordingly, the element of the array will specify the amount of necessary to perform the work of the organization (agent) belonging to the corresponding group. This explains the presence of four parameters (interval_A, interval_B, interval_C and interval_D) in the structure of the Task agent. It is these parameters that are used to determine the volume of work performed by each organization or enterprise related to each of the four groups that make up the RCC.

Also in the integrated structure Agent Task two modules implementing discrete-event approach. The first module is a statechart state diagram consisting, formally, of two states: State_0 - the current execution of the time interval works and the final state $(\bullet)$ - the current jobs are executed. Introduction to consideration of the next element of the collection, which determines information about the time section, occurs when the conditions are met. For this purpose, four variables of the type boolean (A, B, C and D) are defined, initialized as false (false) - this is an indication that the planned volume of work is not performed, when the work is finished, they are defined as true. The second module consists of the blocks of the process modeling library and is the implementation of four-channel QS with the expectation [15]. This module can be viewed from the positions of the flow diagram [16] because the main requirement is fulfilled - only agents of the same type are managed and for this purpose the agent-images described above were introduced.

Now if we go over to the functioning of the simulation model developed, we can conclude that according to the structure, the presented model is an agent model (multiagent model). In addition, it is hybrid, since in addition to the agent approach, there are systemdynamic and discrete-event approaches, that is, all approaches to simulation modeling are integrated in this model [17].

\section{Conclusion}

The article considers a regional building cluster from the standpoint of a decentralized complex system. Proposed and described approaches that allow us to proceed to the consideration of the functioning of this abstraction from the position of a centralized organizational structure. This is realized by introducing image agents and forming a multiphase mass service network, where the four-channel queuing system with expectations is considered as the phase, which allows to take into account the probabilistic structure of business processes arising in the regional construction cluster.

\section{References}


1. D. Anufriev, I. Petrova, O. Shikulskaya Creativity in Intelligent Technologies and Data Science. CIT\&DS 2017. Communications in Computer and Information Science 754, pp (2017)

2. D. Anufriev Proceedings of the II International Scientific and Practical Conference, 58-73 (2010)

3. R. Shannon Systems Simulation: The Art and Science (Prentice-Hall, 1975)

4. R. Shannon Winter Simulation Conference, 7-14 (1998)

5. D. Anufriev MGSU Bull. 12(100) 99-106 (2017)

6. P. Kuehn IEEE Transact. on Communications, 27(1) 113-126 (1979)

7. F. Baskett, K. Chandy, R. Muntz and F. Palacios J. of ACM, 22(2) 248-260 (1975)

8. F. Kaminsky and D. Rumpf Simulation, 29 17-20 (1977)

9. H. Damerdji and M. Nakayama Assoc. Comput. Mach. Trans. Modeling and Comput. Simul., 9 1-30 (1999)

10. L. Schruben Winter Simulation Conference 366-369 (1986).

11. E. Yucesan and L. Schruben Assoc. Comput. Mach. Trans. Modeling and Comput. Simul., 2 82-103 (1992)

12. A. Avramidis and J. Wilson Operations Res., 44 327-346 (1996)

13. R. Teleb and F. Azadivar Operational Res., 72 135-145 (1994)

14. G. Gemot and D. Stark Restructuring Networks: Legacies, Linkages, and Localities in Postsocialism 1-32 (1997)

15. W. Kelton ORSA J. Comput, 6 318-328 (1994)

16. R. Saigent Winter Simulation Conference, 55-64 (1996)

17. J. Donohue Winter Simulation Conference, 200-206 (1994) 